\title{
EFEITO DE DIFERENTES PROCESSAMENTOS SOBRE O TEOR DE ÁCIDO ASCÓRBICO EM SUCO DE LARANJA UTILIZADO NA ELABORAÇÃO DE BOLO, PUDIM E GELÉIA ${ }^{1}$
}

\author{
Patricia Teixeira da SILVA², Maria Lúcia Mendes LOPES², Vera Lúcia VALENTE-MESQUITA**
}

\begin{abstract}
RESUMO
Este estudo teve por objetivo avaliar a estabilidade do ácido ascórbico em suco de laranja cv. "Pêra" utilizado como ingrediente na elaboração de bolos, pudins e geléia. Os bolos e os pudins foram assados em forno convencional e de microondas, e a geléia foi elaborada em tacho aberto. Estas preparações foram submetidas a análises para determinação dos teores de ácido ascórbico e de sólidos solúveis totais, da acidez total titulável e do $\mathrm{pH}$. Os teores de ácido ascórbico em bolos e pudins, quando comparados ao do suco de laranja in natura apresentaram redução de, respectivamente, 76,09\% e 41,76\%, quando assados em forno de microondas, e de $84,21 \%$ e $46,71 \%$, quando assados em forno convencional. A geléia de laranja apresentou redução de $24,86 \%$ no teor de ácido ascórbico em relação ao suco de laranja in natura. A cocção de bolos e pudins realizada em forno de microondas proporcionou maior retenção do ácido ascórbico, quando comparada àquela realizada em forno convencional. Estas preparações apresentaram perda superior à da geléia, elaborada em tacho aberto. Foi observado, também, que os bolos perderam mais ácido ascórbico do que os pudins, em ambos os métodos de cocção avaliados.
\end{abstract}

Palavras-chave: ácido ascórbico, suco de laranja, processamento térmico.

\section{SUMMARY}

EFFECT OF DIFFERENT PROCESSING METHODS ON ASCORBIC ACID CONTENT IN ORANGE JUICE USED TO MAKE CAKES, PUDDINGS AND JELLY. The aim of this study is to analyze the stability of ascorbic acid in orange juice cv. "Pera" used as an ingredient to prepare cakes, puddings and jelly. Cakes and puddings were baked in conventional and microwave ovens and the jelly was prepared in a pan without a lid. Ascorbic acid content, total soluble solids, total tritatable acidity and the pH were determined in all prepared foods. The AA content in cakes and puddings, when compared to the content of orange juice in natura had a reduction of $76.09 \%$ and $41.76 \%$, respectively when baked in a microwave oven and of $84.21 \%$ and $46.71 \%$, when baked in a conventional oven. Orange jelly showed a reduction of $24.76 \%$ in the ascorbic acid content related to orange juice in natura. Preparing cakes and puddings in a microwave oven provided a greater retention of the ascorbic acid, when compared to the conventional oven. These preparations had a higher loss to jelly, made in a pan without a lid. It was also observed that the cakes lost more ascorbic acid than the puddings in both evaluated cooking methods.

Keywords: ascorbic acid, orange juice, thermal process.

\section{1 - INTRODUÇÃO}

Estudos recentes ressaltam a importância de se avaliar o valor nutricional dos alimentos, a fim de se conhecer sua contribuição no suprimento da recomendação diária de nutrientes, bem como a influência do processamento e das tecnologias de preservação na sua composição química [ 13 , $24,26]$. O processamento industrial ou doméstico pode tornar os alimentos mais atraentes ao paladar e aumentar sua vida-de-prateleira. No entanto, podem levar a perdas expressivas, comprometendo a qualidade nutricional do produto final ou da preparação [3, 19].

\footnotetext{
$\overline{{ }^{1} \text { Recebido para publicação em 21/11/2005. Aceito para publicação em }}$ 6/7/2006 (001640). Parte da dissertação intitulada "Estabilidade química e microbiológica do suco de laranja (Citrus sinensis, Osbeck), cultivar Pêra, submetido a diferentes tipos de processamento e condições de estocagem", apresentada ao Programa de Pós-Graduação em Nutrição. Instituto de Nutrição Josué de Castro, Universidade Federal do Rio de Janeiro, 2005

${ }^{2}$ Departamento de Nutrição Básica e Experimental, Instituto de Nutrição, Centro de Ciências da Saúde, Bloco J, $2^{\circ}$ andar, sala 16,

Universidade Federal do Rio de Janeiro, Ilha do Fundão,

CEP 21941-590 Rio de Janeiro (RJ), Brasil

E-mail:valentem@nutricao.ufri.br

* A quem a correspondência deve ser enviada
}

O teor de vitaminas e minerais dos vegetais pode variar dependendo da espécie, do estágio de maturação na época da colheita, de variações genéticas, do manuseio pós-colheita, das condições de estocagem, do processamento e do tipo de preparação elaborada [13, 19]. O conteúdo destes nutrientes no alimento in natura e sua estabilidade podem influenciar a qualidade nutricional do alimento processado [13].

As vitaminas, especialmente a vitamina $\mathrm{C}$, também conhecida como ácido ascórbico (AA), a tiamina e o ácido fólico são sensíveis ao processamento [19]. Devido à instabilidade ao calor, a vitamina $\mathrm{C}$ tem sido empregada como um indicador para medir os efeitos do processamento na retenção de nutrientes [10, 13, 25].

O AA é amplamente distribuído nos produtos de origem vegetal, sendo encontrado, principalmente, em frutas cítricas e hortaliças folhosas [13, 26]. O suco de laranja, considerado fonte de AA [20], é freqüentemente consumido in natura e utilizado em preparações como bolos, pudins, molhos e geléias. Bolos e pudins são ricos em proteínas, glicídios e vitaminas e o consumo destas preparações é bastante difundido entre a população brasileira [4]. A geléia de fruta é utilizada em pães e bolachas ou empregada no recheio de bolo e artigos de confeitaria. No Brasil, as geléias 
apresentam grande importância comercial para a indústria de conservas de frutas. Nos países europeus, este produto assume um papel de destaque, tanto sob o aspecto de consumo quanto de qualidade [22]. O processamento industrial de frutas absorve grande parte da colheita, o que favorece o consumo destas durante o ano todo, além de reduzir o desperdício de alimentos [16].

Os métodos de processamento térmico de alimentos de origem vegetal mais utilizados em nível doméstico são a cocção em vapor, água em ebulição, tacho aberto, forno convencional ou forno de microondas. Tais processos, aplicados isoladamente ou associados a outros processos, por exemplo, mecânicos, podem levar a alterações nas características físicas e na composição química dos alimentos [26].

Alguns trabalhos publicados avaliaram a perda de nutrientes durante o processo de cocção de hortaliças verdes $[11,13,14,23,26]$ e de batatas [5, 12, 18]. BENASSI \& ANTUNES [3] avaliaram, também, a influência de diferentes métodos de cocção de hortaliças na degradação de AA. No entanto, são escassos os estudos que avaliem a estabilidade do AA em sucos de frutas submetidos a diferentes tipos de processamento. As possíveis perdas de AA decorrentes de tais processos precisam ser avaliadas de modo a garantir maior exatidão no planejamento de dietas, bem como na avaliação dietética de indivíduos.

O objetivo deste estudo foi comparar a estabilidade do AA em suco de laranja in natura utilizado como ingrediente na elaboração de bolos e pudins, por diferentes métodos de cocção, bem como de geléia.

\section{2 - MATERIAL E MÉTODOS}

\section{1 - Amostras}

Os ingredientes para a elaboração de bolo, pudim e geléia de laranja foram adquiridos em mercado consumidor da cidade do Rio de Janeiro. O suco de laranja utilizado em todas as preparações foi extraído de laranjas da cultivar "Pêra" e representou a única fonte de AA das receitas.

\section{2 - Elaboração das preparações}

As preparações foram elaboradas no Laboratório de Análise e Processamento de Alimentos (LAPAL) do Instituto de Nutrição Josué de Castro da Universidade Federal do Rio de Janeiro, de acordo com receitas pré-testadas, seguindo as técnicas de elaboração descritas a seguir:

- Bolo de laranja: $200 \mathrm{~mL}$ de suco de laranja in natura foram adicionados à massa de bolo, que continha três ovos inteiros, açúcar refinado (500 g), margarina (200 g), farinha de trigo (500 g), fermento químico (9 g) e misturados em batedeira doméstica. Os bolos foram assados em forno de microondas, em potência alta, por $12 \mathrm{~min}$, e em forno convencional, a $180{ }^{\circ} \mathrm{C}$ por $30 \mathrm{~min}$.

- Pudim de laranja: $200 \mathrm{~mL}$ de suco de laranja in natura foram adicionados à massa de pudim, que continha três ovos inteiros, leite condensado (395 g), margarina (20 g), amido de milho (20 g) e misturados em liquidificador doméstico. Os pudins foram assados em forno de microondas, em potência alta, por $12 \mathrm{~min}$, e em banho-maria, em forno convencional, a $150{ }^{\circ} \mathrm{C}$ por $40 \mathrm{~min}$.

- Geléia de laranja: Açúcar refinado (3 kg) e glicose (200 g) foram adicionados a 2 litros de suco de laranja in natura. Os ingredientes foram aquecidos $\left(100^{\circ} \mathrm{C}\right) \mathrm{em}$ tacho aberto até $55^{\circ} \mathrm{Brix}$, quando foi adicionada pectina $1 \%$ (pré-mix: $10 \mathrm{~g}$ de pectina para $100 \mathrm{~g}$ de açúcar). A geléia foi concentrada até atingir $68^{\circ} \mathrm{Brix}$. O tempo total de cocção foi de 50 min.

\section{3 - Aplicação de processos térmicos e mecânicos em suco de laranja in natura}

Amostras de suco de laranja in natura foram submetidas aos métodos empregados na elaboração de bolo, pudim e geléia: (1) $200 \mathrm{~mL}$ de suco foram aquecidos em forno convencional e em forno de microondas por seis minutos; (2) amostras de $100 \mathrm{~mL}$ de suco foram submetidas à batedura em liquidificador doméstico por até dez minutos; e (3) 2 litros de suco foram adicionados de $70 \%$ de sacarose (p/v) e aquecidos durante 50 min em tacho aberto a fim de simular o preparo da geléia.

\section{4 - Análises químicas}

O teor de AA do suco de laranja in natura, dos sucos submetidos aos processos térmicos e mecânicos, da geléia, dos bolos e dos pudins foi determinado, em triplicata, por titulação com 2,6-diclorofenolindofenol, de acordo com CUNNIFF [6], método número 967.21, com as modificações propostas por BENASSI \& ANTUNES [2]. A determinação do teor de AA do bolo e do pudim foi feita com base na análise de 100 g da preparação diluída em água destilada e filtrada; enquanto que para a geléia, a diluição foi realizada em ácido oxálico 1\%, de acordo com o protocolo padrão da análise de Tillmans.

A acidez total titulável (ATT), expressa em g de ácido cítrico anidro/100 g de suco, foi determinada, em triplicata, por titulação com 0,1 N de $\mathrm{NaOH}$, de acordo com CUNNIFF [6], método 942.15A.

\section{5 - Análises físico-químicas}

$\mathrm{O}$ pH foi determinado em pHmetro digital (Incibrás, Brasil) com compensação automática de temperatura. A quantificação dos sólidos solúveis totais (SST), expressa em ${ }^{\circ}$ Brix, foi realizada por meio de leitura direta em refratômetro (Eppendorf 2763, Brasil). Estas análises foram realizadas de acordo com CUNNIFF [6], métodos 983.17 e 942.15B, respectivamente.

\section{6 - Análises estatísticas}

Os resultados foram submetidos à análise de variância ANOVA, seguida pelo teste de Tukey ou à análise de regressão [15]. A significância estatística foi estabelecida ao nível 
de $5 \%$ de probabilidade. O software estatístico utilizado foi o Excel 2000 (Microsoft Corporation, EUA).

\section{3 - RESULTADOS E DISCUSSÃO}

A Tabela 1 apresenta o teor médio de AA do suco de laranja in natura e de bolos e pudins assados em fornos de microondas e convencional. Foi observada diferença significativa no teor médio de AA entre as preparações elaboradas pelos diferentes métodos de cocção. A redução do teor de AA no bolo e no pudim assados em forno de microondas foi significativamente menor do que a das mesmas preparações assadas em forno convencional. ZHANG \& HAMAUZU [26] observaram que o percentual de redução do teor de AA em brócolis cozido em forno de microondas foi menor do que o coccionado em água em ebulição. HOWARD et al. [13], ao estudarem a influência do processamento térmico no teor de AA e $\beta$-caroteno em hortaliças folhosas, observaram que a cocção em forno de microondas não afetou a concentração de AA. ERDMAN \& KLEIN [8] observaram que a quantidade de água adicionada e o tempo de cocção interferiram mais na estabilidade do AA do que a fonte de energia ou o tipo de cocção utilizado; enquanto que TAPADIA, ARYA \& ROHINI DAVI [23] atribuíram as perdas de vitamina $\mathrm{C}$ em vegetais ao processo de cocção.

A cocção em forno de microondas permite alcançar temperaturas superiores à do forno convencional o que, teoricamente, resultaria em perda mais expressiva de AA por degradação térmica. No entanto, esta redução pode ser minimizada pelo fato de a cocção em microondas permitir um aquecimento mais uniforme e mais rápido do alimento, quando comparado ao cozimento convencional em que a condução do calor se processa de maneira mais lenta [10]. BENASSI \& ANTUNES [3] estudaram a cinética de degradação da vitamina $\mathrm{C}$ no cozimento doméstico de vegetais e observaram que a solubilização desta vitamina é minimizada no processo de cocção em forno de microondas, o que pode levar a maior retenção de AA quando comparado à cocção em água. Além disso, o menor tempo empregado na cocção em forno de microondas pode ter contribuído para minimizar a perda do AA.

O estudo realizado com o suco de laranja in natura submetido aos diferentes métodos de cocção demonstrou que há diferença significativa entre o teor médio de AA deste suco e o daqueles que foram aquecidos em fornos de microondas e convencional. Do mesmo modo que o teor de AA, o teor de SST no suco aquecido em forno de microondas foi significativamente maior do que no suco in natura (Tabela 2). Este aumento provavelmente se deve à concentração por desidratação. Desta forma foi feita a reconstituição do suco aquecido em forno de microondas, por adição de água, até atingir o valor inicial de SST. Após reconstituição, a determinação do teor de AA não indicou diferença significativa em relação ao do suco in natura. Estes resultados poderiam também justificar menor redução no teor de AA nas preparações assadas em forno de microondas.

A Figura 1 apresenta o teor médio de AA em suco de laranja in natura submetido à agitação mecânica por até 10 min. Foi observada diferença significativa entre o teor médio de AA no suco in natura e nas amostras liquidificadas.

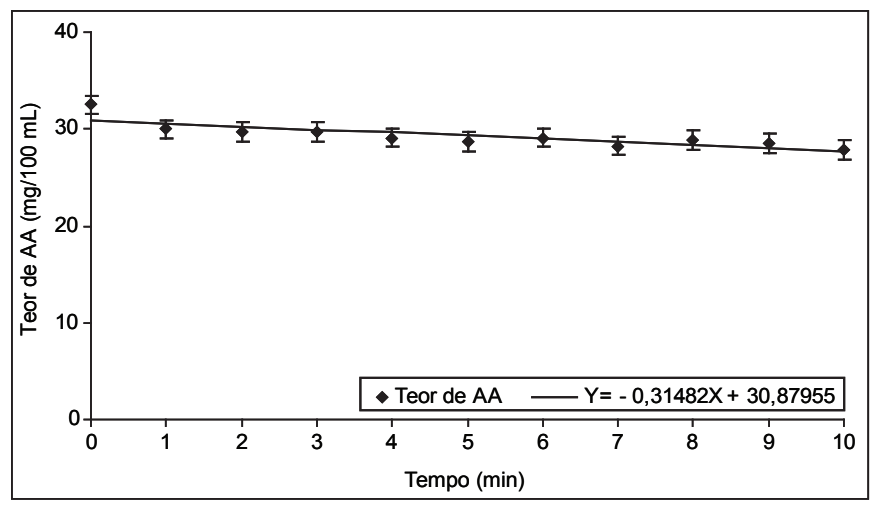

FIGURA 1 - Teor médio de ácido ascórbico (AA) em suco de laranja in natura submetido à agitação mecânica em liquidificador doméstico.

TABELA 1 - Teor médio de ácido ascórbico (AA) em suco de laranja in natura e em bolos e pudins, assados em forno de microondas e convencional, contendo este suco como ingrediente.

\begin{tabular}{lcccccc} 
& Suco de laranja in natura & \multicolumn{2}{c}{ Bolo } & & \multicolumn{2}{c}{ Pudim } \\
\cline { 2 - 4 } & & Microondas & Forno Convencional & & Microondas & Forno Convencional \\
\hline Teor de AA $(\mathrm{mg})$ & $83,78^{a^{* *}}$ & $20,03^{\mathrm{b} * *}$ & $13,23^{\text {c**}}$ & & $48,79^{\mathrm{d} * *}$ & $44,62^{\mathrm{e} * *}$ \\
Redução de AA $(\%)$ & - & 76,09 & 84,21 & & 41,76 & 53,29 \\
\hline
\end{tabular}

Médias em uma mesma linha seguidas de mesma letra, não diferem entre si pelo teste de Tukey a 5\% de probabilidade. ${ }^{*}$ Teor de AA em 200 mL; e **Teor de AA por peso total da preparação.

TABELA 2 - Teores médios de ácido ascórbico (AA) e de sólidos solúveis totais (SST) em suco de laranja in natura e aquecido em fornos de microondas e convencional.

\begin{tabular}{|c|c|c|c|c|}
\hline & \multicolumn{4}{|c|}{ Suco de laranja } \\
\hline & In natura & $\begin{array}{l}\text { Aquecido em forno de } \\
\text { microondas }\end{array}$ & $\begin{array}{c}\text { Aquecido em forno de } \\
\text { microondas com reconstituição } \\
\text { do teor de SST }\end{array}$ & $\begin{array}{l}\text { Aquecido em forno } \\
\text { convencional }\end{array}$ \\
\hline $\mathrm{AA}(\mathrm{mg} / 100 \mathrm{~mL})$ & $28,63^{a}$ & $38,03^{b}$ & $28,60^{a}$ & $27,99^{c}$ \\
\hline SST $\left({ }^{\circ}\right.$ Brix $)$ & $10^{\mathrm{a}}$ & $13^{b}$ & $10^{a}$ & $10^{a}$ \\
\hline
\end{tabular}

Médias seguidas de mesma letra, em uma mesma linha, não diferem entre si pelo teste de Tukey a 5\% de probabilidade. 
O teor médio de AA variou de $32,49 \mathrm{mg} / 100 \mathrm{~mL}$ para o suco não liquidificado a $27,85 \mathrm{mg} / 100 \mathrm{~mL}$ para aquele submetido a dez minutos de agitação. O teor de SST permaneceu constante, 11,5 ${ }^{\circ}$ Brix, em todos os tempos de liquidificação. Este resultado demonstra o efeito da agitação mecânica na estabilidade do AA. Tal processo, empregado na elaboração de bolos e pudins, pode, também, ter contribuído para a redução do teor de AA, provavelmente pela incorporação de ar, com conseqüente oxidação desta vitamina.

De acordo com ALBUQUERQUE [1], as geléias apresentam, em geral, pH e teor de SST em torno de 3,1 e $65{ }^{\circ}$ Brix, respectivamente. O suco de laranja utilizado no processamento da geléia apresentou $48,55 \mathrm{mg} / 100 \mathrm{~mL}$ de AA, $0,96 \mathrm{~g} / 100 \mathrm{~mL}$ de ATT, $11^{\circ} \mathrm{Brix}$ e pH 3,4. Estes resultados são semelhantes aos descritos por SILVA et al. [21]. O suco de laranja in natura utilizado na elaboração de geléia apresentou $\mathrm{pH}$ mais elevado do que o considerado ideal à formação de gel, que deve estar na faixa de 3,0 a 3,2 [1]. No entanto, testes preliminares demonstraram que não seria necessário ajustar o $\mathrm{pH}$ da matéria-prima empregada.

A Tabela 3 apresenta os dados de AA e de SST em suco de laranja in natura e adicionado de sacarose e em geléia de laranja. Embora o teor médio de AA da geléia tenha sido significativamente inferior ao do suco de laranja in natura, uma retenção de aproximadamente $75 \%$ desta vitamina foi verificada no produto final. Este resultado é inferior ao relatado por MÉLO, LIMA \& NASCIMENTO [16] que, ao avaliarem a estabilidade do AA na elaboração de geléia a partir da mistura dos sucos de pitanga e acerola, observaram um percentual de retenção de aproximadamente $93 \%$.

Os teores médios de AA no suco adicionado de sacarose antes e após o tratamento térmico em tacho aberto corresponderam a $68,20 \%$ e $62,68 \%$, respectivamente, do teor de AA do suco in natura. Os resultados demonstram que tanto a adição de sacarose quanto o tratamento térmico influenciaram significativamente o teor de AA destas amostras.

A Tabela 4 apresenta o teor médio de AA em bolo, pudim e geléia e o percentual da ingestão dietética de referência - IDR [7] para homens adultos suprido pelo consumo de 100 g de cada preparação. Embora a geléia apresente teor percentual de AA superior ao das demais preparações, a porção consumida é, geralmente, menor. Desta forma, fatias pequenas ( $100 \mathrm{~g}$ ) de bolo e de pudim suprem $2,0 \% \mathrm{e}$ $8,5 \%$ da IDR, respectivamente; enquanto que, uma porção de geléia (20 g) supre $8,1 \%$ da IDR [7]. Estes resultados demonstram que bolo, pudim e geléia de laranja não podem ser considerados alimentos fontes de vitamina $\mathrm{C}$, uma vez que seria necessária a ingestão de elevada quantidade destas preparações para atingir a IDR, valor este que poderia ser alcançado com a ingestão de apenas um copo (200 mL) de suco de laranja.

TABELA 4 - Teor médio de ácido ascórbico (AA) de bolo, pudim e geléia de laranja e o percentual da ingestão dietética de referência (IDR $=90 \mathrm{mg})^{*}$, suprido pelo consumo de $100 \mathrm{~g}$ de cada preparação.

\begin{tabular}{lcc}
\hline \multicolumn{1}{c}{ Preparação } & $\begin{array}{c}\text { Teor de AA } \\
(\mathbf{m g} / \mathbf{1 0 0} \mathbf{~ m L})\end{array}$ & $\begin{array}{c}\text { Percentual que } \\
\text { supre da IDR }\end{array}$ \\
\hline Suco de Laranja & 45,22 & 50,24 \\
Bolo & 1,80 & 2,00 \\
Pudim & 7,66 & 8,51 \\
Geléia & 36,48 & 40,53 \\
\hline
\end{tabular}

*IDR: Ingestão dietética de referência [7]; e ${ }^{* *}$ Média calculada a partir dos resultados para suco de laranja e para as preparações assadas em forno convencional e em forno de microondas.

Ao estabelecer uma comparação entre os teores de AA obtidos no presente estudo e aqueles descritos em tabelas nacionais de composição química de alimentos, foram encontrados somente os dados para geléia de laranja em uma tabela [9], cujo valor descrito corresponde a $27,63 \%$ do apresentado neste estudo. PINHEIRO et al. [17] apresentam os dados para geléia de fruta, sem, no entanto, especificar qual a fruta utilizada em sua elaboração. Nesta mesma tabela, consta o teor de AA de bolo e pudim sem, no entanto, mencionar se estes continham suco de laranja entre os seus ingredientes. A escassez de dados sobre o conteúdo de vitaminas em alimentos processados dificulta o trabalho dos profissionais que atuam na avaliação e no planejamento de dietas e ratifica a necessidade de estudos relacionados às perdas de nutrientes durante o preparo de alimentos.

\section{4 - CONCLUSÕES}

A geléia de laranja apresentou teor de AA maior quando comparado ao de bolos e pudins, evidenciando a sensibilidade desta vitamina ao tipo de processamento a que as preparações foram submetidas.

O percentual de retenção de AA no bolo e no pudim assados em forno de microondas foi maior do que o das mesmas preparações assadas em forno convencional.

O processo de liquidificação do suco de laranja in natura reduziu significativamente o teor de AA, demonstrando que não só o processamento térmico, mas também

TABELA 3 - Teor médio de ácido ascórbico (AA) e de sólidos solúveis totais (SST) em suco de laranja in natura, em suco de laranja adicionado de sacarose antes e depois de tratamento térmico e em geléia de laranja.

\begin{tabular}{|c|c|c|c|c|}
\hline & \multirow[t]{2}{*}{ Suco in natura } & \multirow[t]{2}{*}{ Geléia } & \multicolumn{2}{|c|}{ Suco com sacarose $(70 \% \mathrm{p} / \mathrm{v})$} \\
\hline & & & Antes do aquecimento & Após aquecimento \\
\hline $\mathrm{AA}(\mathrm{mg} / 100 \mathrm{~mL})$ & $48,55^{\mathrm{a}}$ & $36,48^{\mathrm{b}}$ & $33,09^{c}$ & $30,43^{d}$ \\
\hline SST ( ${ }^{\circ}$ Brix $)$ & 11 & 65 & 47 & 47 \\
\hline
\end{tabular}

Médias em uma mesma linha seguidas de mesma letra, não diferem entre si pelo teste de Tukey a 5\% de probabilidade. 
a agitação mecânica podem influenciar o valor nutricional das preparações.

Os resultados do presente estudo suprem uma demanda por parte dos profissionais da área de alimentos no que se refere a dados sobre o conteúdo de vitamina $\mathrm{C}$ em alimentos processados.

\section{5 - REFERÊNCIAS BIBLIOGRÁFICAS}

[1] ALBUQUERQUE, J. P. Fatores que influem no processamento de geléias e geleiadas de frutas. Boletim SBCTA, v. 31, n. 1, p. 62-67, 1997.

[2] BENASSI, M. T.; ANTUNES, A. J. A comparison of metaphosphoric and oxalic acids as extractants solutions for the determination of vitamin $\mathrm{C}$ in selected vegetables. Arquivos de Biologia e Tecnologia, v. 31 , n. 4, p. 507-513, 1988.

[3] BENASSI, M. T.; ANTUNES, A. J. Cinética de degradação de vitamina $C$ no cozimento doméstico de vegetais. In: CONGRESSO BRASILEIRO DE CIÊNCIA E TECNOLOGIA DE ALIMENTOS, XVIII, 2002, Porto Alegre, Anais do XVIII Congresso Brasileiro de Ciência e Tecnologia de Alimentos, Rio Grande do Sul, 2002, cd-rom.

[4] BRASIL Ministério da Saúde. Estudo multicêntrico sobre consumo alimentar, 1997.Disponível em:<http:// dtr2001.saude.gov.br/bvs/publicacoes/cadernosespecial. pdf $>$. Acesso em: 15 jan. 2005.

[5] CHALOM, S.; ELREZZI, E.; PEÑA, P.; ASTIARSARÁN, I.; BELLO, J. Composition of sulfited potatoes: comparision with fresh and frozen potatoes. Plant Foods for Human Nutrition, v. 47, n. 2, p. 133-138, 1995.

[6] CUNNIFF, P. Fruits and fruit products. In: Official Methods of Analysis of AOAC International. Arlington: Virgini A, USA, 1995. Cap. 37, v. 2, p. 7,10.

[7] DIETARY REFERENCE INTAKES: Applications in Dietary Assessment 2001. National Academy Press 71-72. Disponível em: <http://books.nap.edu/books/0309071836/ html/289.html>. Acesso em: $17 \mathrm{dez} .2002$.

[8] ERDMAN, J. W. Jr., KLEIN, B. P. Harvesting, processing, and cooking influences on vitamin $\mathrm{C}$ in foods. In: Ascorbic Acid Chemistry, Metabolism and Uses. Eds. SEIB, P. A. and TOLBERT, B. M. Washington, D.C., 1982.

[9] FRANCO, G. Tabela de Composição Química dos Alimentos. 9 ${ }^{a}$ edição, Ed. Atheneu, São Paulo, 1999.

[10] GESTER, H. Vitamin losses with microwave cooking. Food Sciences and Nutrition, v. 42F, p. 173-181, 1989.

[11] GIANNAKOUROU, M. C.; TAOUKIS, P. S. Kinetic modeling of vitamin $\mathrm{C}$ loss in frozen green vegetables under variable storage conditions. Food Chemistry, v. 83, n.1, p. 33-41, 2003.

[12] HAASE, N. U.; WEBER, L. Ascorbic acid losses during processing of French fries and potato chips. Journal of Food Engineering, v. 56, p. 207-209, 2003.

[13] HOWARD, L. A.; WONG, A. D.; PERRY, A. K.; KLEIN, B. P. $\beta$-carotene and ascorbic acid retention in fresh and processed vegetables. Journal of Food Science, v. 64, n. 5, p. 929-936, 1999.
[14] INYANG, U. E.; IKE, C. I. Effect of blanching, dehydration method and temperature on the ascorbic acid, colour, sliminess and other constituents of okra fruit. International Journal of Food Science and Nutrition, v. 49, p. 125-130, 1998.

[15] LAPPONI, J. C. Estatística usando Excel. São Paulo: Lapponi Treinamento e Editora, 2000.

[16] MÉLO, E. A.; LIMA, V. L. A. G.; NASCIMENTO, P. P. Formulação e avaliação físico-química e sensorial de geléia mista de pitanga (Eugenia uniflora L.) e acerola (Malpighia sp). Boletim CEPPA, v. 17, n. 1, p. 33-44, 1999.

[17] PINHEIRO, A. B. V.; LACERDA, M. E. A.; BENZECRY, E. H.; GOMES, M. C. S.; COSTA, V. M. Tabela de Avaliação de Consumo Alimentar em Medidas Caseiras. $4^{\mathrm{a}}$ edição, editora Atheneu, Rio de Janeiro, 2001.

[18] REDMOND, G. A.; DECAZES, A. M.; GORMLEY, T. R.; BUTLER, F. The vitamin C status of freeze-chilled mashed potato. Journal of Food Engineering, v. 56, p. 219-221, 2003.

[19] REDY, M. B.; LOVE, M. The impact of food processing on the nutritional quality of vitamins and minerals. Impact of Processing on Food Safety, v. 459, p. 99-106, 1999.

[20] SHAW, P. E.; MOSHONAS, M. G. Ascorbic Acid Retention in Orange Juice Stored under Simulated Consumer Home Conditions. Journal of Food Science, Chicago, v. 56, n. 3, p. 867-868, 1991.

[21] SILVA, P. T.; PEREIRA, C. Q.; ALMEIDA, A. S.; MIGUEL, M. A. L.; LOPES, M. L. M.; VALENTE-MESQUITA, V. L. Efeito da temperatura e do tempo de estocagem sobre o teor de ácido ascórbico e a estabilidade microbiológica de suco de laranja 'Pêra'. In: SIMPÓSIO LATINO AMERICANO DE CIÊNCIA DE ALIMENTOS, V, 2003, São Paulo. Anais... São Paulo: Unicamp, 2003, cd-rom.

[22] SOLER, M. P. Industrialização de geléias. Campinas: ITAL [Manual Teórico n. 17], 1991.

[23] TAPADIA, S. B.; ARYA, A. B., ROHINI DEVI, P. Vitamin C contents of processed vegetables. Journal of Food Science and Technology, v. 32, n. 6, p. 513-515, 1995.

[24] TUDELA, J. A.; ESPÍN, J. C.; GIL, M. I. Vitamin C retention in fresh-cut potatoes. Postharvest Biology and Technology, v. 26, p. 75-84, 2002.

[25] VANDERLISE, J. T.; HIGGS, D. J.; HAYES, J.M.; BLOCK, G. Ascorbic acid and dehydroascorbic acid content of food-as-eaten. Journal of Food Composition and Analisys, v. 3, p. 105-118, 1990.

[26] ZHANG, D; HAMAUZU, Y. Phenolics, ascorbic acid, carotenoids and antioxidant activity of broccoli and their changes during conventional and microwave cooking. Food Chemistry, v. 88, p. 503-509, 2004.

\section{6 - AGRADECIMENTOS}

Os autores agradecem à FAPERJ e FUJB pelo apoio financeiro, que proporcionou a realização deste trabalho. 